\title{
Successful Laparoscopy-assisted Repair of a Rectovaginal Fistula after Low Anterior Resection for Rectal Cancer: A Report of Two Cases
}

Hiroyuki Ohta ( $\sim$ hohta@belle.shiga-med.ac.jp)

Higashi-Ohmi General Medical Center https://orcid.org/0000-0002-3318-9263

Kyozo Hashimoto

Hashimioto Clinic

Tomoyuki Mizukuro

Nagaokakyo Hospital

Byonggu An

National Hospital Organisation Higashiomi General Medical Center: Kokuritsu Byoin Kiko Higashiomi

Sogo Iryo Center

Yumi Zen

National Hospital Organisation Higashiomi General Medical Center: Kokuritsu Byoin Kiko Higashiomi

Sogo Iryo Center

Masatsugu Kojima

Shiga University of Medical Science: Shiga Ika Daigaku

Tomoyuki Ueki

Shiga University of Medical Science: Shiga Ika Daigaku

Toru Miyake

Shiga University of Medical Science: Shiga Ika Daigaku

\section{Yusuke Nishina}

National Hospital Organisation Higashiomi General Medical Center: Kokuritsu Byoin Kiko Higashiomi Sogo Iryo Center

\section{Yoshitaka Terada}

National Hospital Organisation Higashiomi General Medical Center: Kokuritsu Byoin Kiko Higashiomi Sogo Iryo Center

\section{Naomi Kitamura}

National Hospital Organisation Higashiomi General Medical Center: Kokuritsu Byoin Kiko Higashiomi Sogo Iryo Center

Hiroya Akabori

National Hospital Organisation Higashiomi General Medical Center: Kokuritsu Byoin Kiko Higashiomi Sogo Iryo Center

Mitsuhiro Fujino 
Nagaokakyo Hospital

\section{Tomoharu Shimizu}

Shiga University of Medical Science: Shiga Ika Daigaku

Masaji Tani

Shiga University of Medical Science: Shiga Ika Daigaku

\section{Eiji Mekata}

National Hospital Organisation Higashiomi General Medical Center: Kokuritsu Byoin Kiko Higashiomi Sogo Iryo Center

\section{Case report}

Keywords: rectovaginal fistula, rectal cancer, low anterior resection, double-stapling technique

Posted Date: October 23rd, 2020

DOI: https://doi.org/10.21203/rs.3.rs-94548/v1

License: (c) (i) This work is licensed under a Creative Commons Attribution 4.0 International License. Read Full License 


\section{Abstract}

Background: A rectovaginal fistula (RVF) after low anterior resection for rectal cancer is troublesome and refractory. Although various surgical procedures have been previously described, no definitive procedure has shown satisfactory outcomes. We present two consecutive Japanese patients who underwent successful surgery for a RVF after low anterior resection.

Case presentation: The patients were a 61-year-old woman and a 64-year-old woman. They were admitted to our hospital with a chief complaint of fecal discharge from the vagina after low anterior resection using the double-stapling technique for rectal cancer. They were diagnosed with RVF. Local surgical procedures, including diverting ileostomy, were unsuccessful in previous hospitals. Therefore, we performed laparoscopy-assisted repair of the RVF. In both the patients, laparoscopically robust pelvic adhesions were dissected, and the sigmoid colon was transected just before the RVF. Thereafter, in combination with a perineal approach, the rectum along with a previous anastomosis and fistula were completely removed. Surgeries were completed after vaginal repair, redo coloanal anastomosis, and interposition of the dissected connective tissue. In both the patients, the postoperative courses were uneventful. They have complained of neither recurrence of any RVF nor fecal incontinence 1 year and 10 months respectively after diverting stoma closure.

Conclusions: A laparoscopy-assisted procedure with reanastomosis and interposition of the perineal connective tissue can be an effective treatment for an RVF after low anterior resection for rectal cancer.

\section{Background}

The rectovaginal fistula (RVF), which is defined as the presence of an orifice between the rectum and the vagina, results in involuntary leakage of intestinal gas and stool from the fistula. Patients suffer from constant discomfort and psychological distress which affect their quality of life. The most common cause of RVF in developing countries is obstetric vaginal trauma (1). Low anterior resection (LAR) for rectal cancer using the double-stapling technique (DST) occasionally leads to RVF in developed countries, with a reported incidence of $1.0-9.9 \%$ (2)-(5).

Various surgical techniques such as temporary diverting stoma, endorectal advancement flap (6), and Gracilis muscle interpositions (7) have been previously described. However, the optimal surgical treatment of RVF remains controversial (8). Here, we report two patients who underwent successful laparoscopy-assisted repair of RVF after LAR for rectal cancer.

\section{Case Presentation}

\section{Patient 1}

A 61-year-old Japanese woman was admitted to our hospital with a chief complaint of fecal discharge from the vagina. She received laparoscopic LAR for early stage rectal cancer 2 years and 9 months ago in 
another hospital. Surgical site drainage and diverting ileostomy were also performed due to anastomotic leakage on the 4th day after surgery. Anastomotic leakage seemed to have healed 4 months after ileostomy; therefore, stoma reversal was performed. Immediately after surgery, she noticed fecal discharge from the vagina and was diagnosed with an RVF. Despite a second diverting ileostomy and transvaginal repair, the RVF had not cured. Colonoscopic examination revealed a rectovaginal fistula measuring $10 \mathrm{~mm}$ in diameter, situated $3 \mathrm{~cm}$ from the anal verge, on the oral side (Fig. 1a). Gastrografin enema showed influx of contrast medium into the vagina (Fig. 1b). Magnetic resonance imaging showed a fistula between the rectum and the vagina. Pelvic abscesses or recurrent tumors were not observed (Fig. 2).

Local minor surgical procedures, including diverting ileostomy, were unsuccessful; therefore, we thought that a major procedure with coloanal reanastomosis was necessary. Laparoscopy-assisted repair of the RVF was performed under general anesthesia with the patient in the lithotomy position. The procedure consists of three steps.

The first step involves carefully dissecting the sigmoid colon and retroperitoneal tissue, avoiding injury to the mesocolonic vessels. Mobilization of the splenic flexure was then performed to decrease tension on the coloanal anastomosis. In the pelvic cavity, the sigmoid colon was detached from the anterior surface of the sacrum, and adhesions around the uterus next to the RVF were removed. Intracorporal sigmoid colon transection was performed with a linear stapler on the oral side of the RVF.

In the second step, a transverse skin incision was made on the perineum. The connective tissues between the rectum and the vagina were dissected up to a depth of approximately $6 \mathrm{~cm}$ from the skin level. During this dissection, the fistula was released (Fig. 3) and the surrounding infectious tissue was removed completely. The fibrous connective tissues around the RVF were exposed clearly on each of lateral sides. Additionally, using the laparoscopic route, the sigmoid colon, including the previous anastomosis was transected and removed at the level of the superior border of the puborectal sling (Fig. 4).

In the third step, the defect on the posterior vaginal wall was closed using interrupted $3-0$ polyglactin sutures. Subsequently, redo coloanal anastomosis was performed by hand-sewn. Furthermore, firm longitudinal suturing of the innermost portion of the dissected fibrous tissues around the RVF on each of the lateral sides was performed by making 3 stitches using $3-0$ polyglactin (Fig. 5). The dissected fibrous tissues were repaired in 4 layers from bottom to top, by making 3 stitches per layer, and the repaired tissues were interposed between previously anastomosed vagina and colon (Fig. 3b). The operation lasted for 691 minutes and the estimated blood loss was $795 \mathrm{~g}$.

The postoperative clinical course was good and the patient was discharged from the hospital on the 16th day following surgery. Stoma closure was performed 11 months after surgery. She had no recurrence of RVF and no complaint of fecal incontinence one year after diverting stoma closure.

\section{Patient 2}


A 64-year-old Japanese woman was referred to our hospital with a chief complaint of fecal discharge from the vagina. She received laparoscopic LAR for early stage rectal cancer 1 year prior to her referral. On the 3rd day after surgery, she complained of fecal discharge and flatulence from the vagina, and she was diagnosed with RVF. Diverting ileostomy was performed 8 months prior to her referral but the RVF had not cured. A fistula measuring approximately $2 \mathrm{~mm}$ in size, was found $4.5 \mathrm{~cm}$ from the anal verge, on the oral side.

Laparoscopy-assisted repair of RVF was performed in the same way as the previous patient. The operation lasted 615 minutes and the estimated blood loss was $700 \mathrm{~g}$. The postoperative clinical course was uneventful. She had no recurrence of RVF and did not complain of fecal incontinence 10 months after stoma closure.

\section{Discussion}

Anastomotic healing disturbances following LAR occasionally lead to RVF. Possible etiologies of RVF include the following: stapling of the vagina during anastomosis, spontaneous drainage of a pelvic abscess through the vagina, and devascularization of the vagina near the anastomosis (9). There are two approaches for managing RVFs: local conservative procedures (rectal or vaginal advancement flaps, fistula plug, fibrin glue, and diverting stoma only) and more aggressive procedures (Gracilis or Martius muscle interposition and abdominal surgery with colorectal or coloanal reanastomosis) (10). Recent reports suggest that aggressive surgical treatment of RVF, including early construction of a diverting stoma and abdominal surgery with redo anastomosis, might result in high success rates (10)(11). However, abdominal surgery with redo anastomosis could be technically troublesome because of previous anastomotic disturbance and consequent severe pelvic adhesion. In our experience, laparoscopy was helpful for adequate dissection of the vagina and rectum, which is a complicated procedure in patients with previous surgery of the pelvic cavity. To the best of our knowledge, there are no reports of laparoscopic treatment for RVF after LAR in English literature.

A unique feature of our procedure is the repair and interposition of the dissected connective tissue, probably including perineal bodies, between the anastomosed vagina and colon. Tissue interposition approaches, such as Gracilis or Martius muscle interpositions, are intended to interpose well-vascularized tissue between suture lines and contribute to wound healing (7). Our method has the advantage of avoiding a wide skin incision and motility disturbance compared to muscle interposition approaches.

In our case, the location of the RVF was low and near the vaginal fourchette. Therefore, a perineal approach with fistulectomy and interposition of the dissected perineal connective tissue was possible. When the location of an RVF is high, the abdominal approach with fistulectomy and colorectal reanastomosis could have been sufficient.

There is no conclusive evidence of the long-term efficacy of our method. Therefore, similar cases should be followed for a longer time after surgery. 


\section{Conclusion}

We successfully carried out two consecutive surgeries in patients with RVF after LAR for rectal cancer. Laparoscopy-assisted procedures with reanastomosis and interposition of the dissected perineal connective tissue may improve outcomes in patients with RVF.

\section{Abbreviations}

RVF: Rectovaginal fistula

LAR: low anterior resection

DST: double stapling technique

\section{Declarations}

\section{Ethics approval and consent to participate}

The collection of patient's clinical data was approved by the Ethics Committee of the Higashi-Ohmi General Medical Center and performed in accordance with the Declaration of Helsinki (approved IRB number: R2-22).

\section{Consent for publication}

Informed consent was obtained from the patients for this publication.

\section{Availability of data and material}

We will share our data and materials on demand.

\section{Funding}

The authors declare that no funding was received for this study.

\section{Authors' contributions}

$\mathrm{HO}$ conceived the case presentation and drafted the manuscript. $\mathrm{KH}, \mathrm{TM}, \mathrm{BA}, \mathrm{YZ}$, and $\mathrm{MK}$ participated in patient treatment. TU, TM, YN, YT, NK, HA, MF, TS, MT, and EM helped to draft the manuscript. All authors have read and approved the final manuscript. 


\section{Acknowledgements}

The authors thank all the individuals who contributed to this work.

\section{Competing interests}

The authors declare that they have no competing interests.

\section{References}

1. Cook RJ, Dickens BM, Syed S. Obstetric fistula: The challenge to human rights. Int J Gynecol Obstet. 2004;87:72-7.

2. Baran JJ, Goldstein SD, Resnik AM. The double-staple technique in colorectal anastomoses: a critical review. Am Surg. 1992;58:270-2.

3. Zheng H, Guo T, Wu Y, Li C, Cai S, Liu F, Xu Y. Rectovaginal fistula after low anterior resection in Chinese patients with colorectal cancer. Oncotarget. 2017;8:73123-32.

4. Watanabe J, Ota M, Kawaguchi D, Shima H, Kaida S, Osada S, Kamimukai N, Kamiya N, Ishibe A, Watanabe K, Matsuyama R, Akiyama H, Ichikawa Y, Oba M, Endo I. Incidence and risk factors for rectovaginal fistula after low anterior resection for rectal cancer. 2015;30:1659-66.

5. Kosugi C, Saito N, Kimata Y, Ono M, Sugito M, Ito M, Sato K, Koda K, Miyazaki M. Rectovaginal fistulas after rectal cancer surgery: Incidence and operative repair by gluteal-fold flap repair. 2005;137;329-36.

6. Sonoda T, Hull T, Piedmonte MR, Fazio VW. Outcomes of Primary Repair of Anorectal and Rectovaginal Fistulas Using the Endorectal Advancement Flap. Dis Colon Rectum. 2002;45:1622-8.

7. Lefèvre JH, Bretagnol F, Maggiori L, Alves A, Ferron M, Panis Y. Operative results and quality of life after gracilis muscle transposition for recurrent rectovaginal fistula. Dis Colon Rectum. 2009;52:1290-5.

8. Pinto RA, Peterson T V., Shawki S, Davila GW, Wexner SD. Are there predictors of outcome following rectovaginal fistula repair? Dis Colon Rectum. 2010;53:1240-7.

9. Rex JC, Khubchandani IT. Rectovaginal fistula. Dis Colon Rectum. 1992;35:354-6.

10. Corte H, Maggiori L, Treton X, Lefevre JH, Ferron M, Panis Y. Rectovaginal Fistula. Ann Surg. 2015;262:855-61.

11. Ryoo S, Oh H, Ha H, Han EC, Kwon Y, Song I, Moon SH, Choe EK, Park KJ. Outcomes of surgical treatments for rectovaginal fistula and prognostic factors for successful closure: a single-center tertiary hospital experiences. Ann Surg Treat Res. 2019;97:149-56.

\section{Figures}



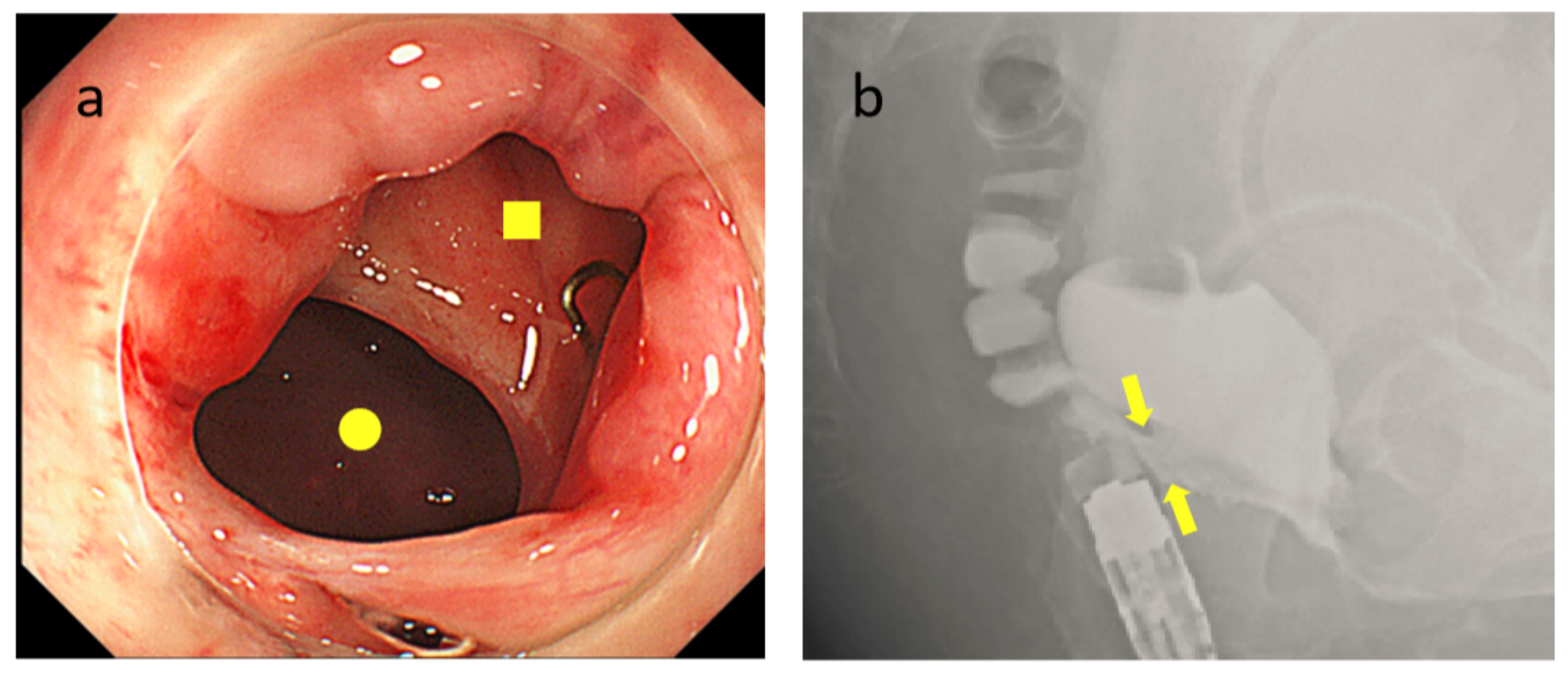

Figure 1

a: Colonoscopic examination demonstrates a rectovaginal fistula $10 \mathrm{~mm}$ in diameter, located $3 \mathrm{~cm}$ from the anal verge, on the oral side. b: Gastrografin enema shows influx of the contrast medium to the vagina through a fistula (arrows). 


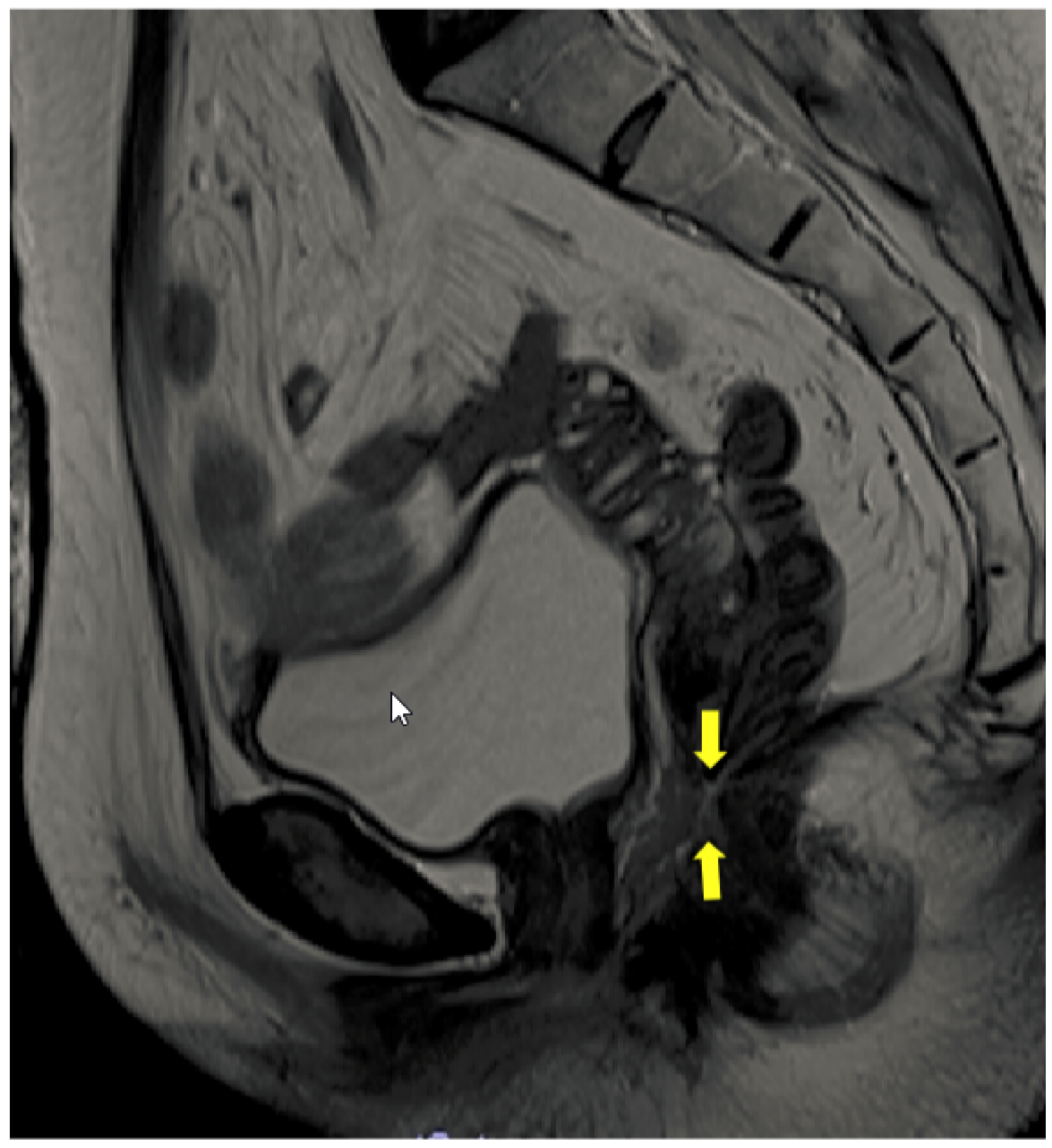

Figure 2

Magnetic resonance imaging shows a fistula between the rectum and vagina (arrows). No pelvic abscesses or recurrent tumors are seen. 


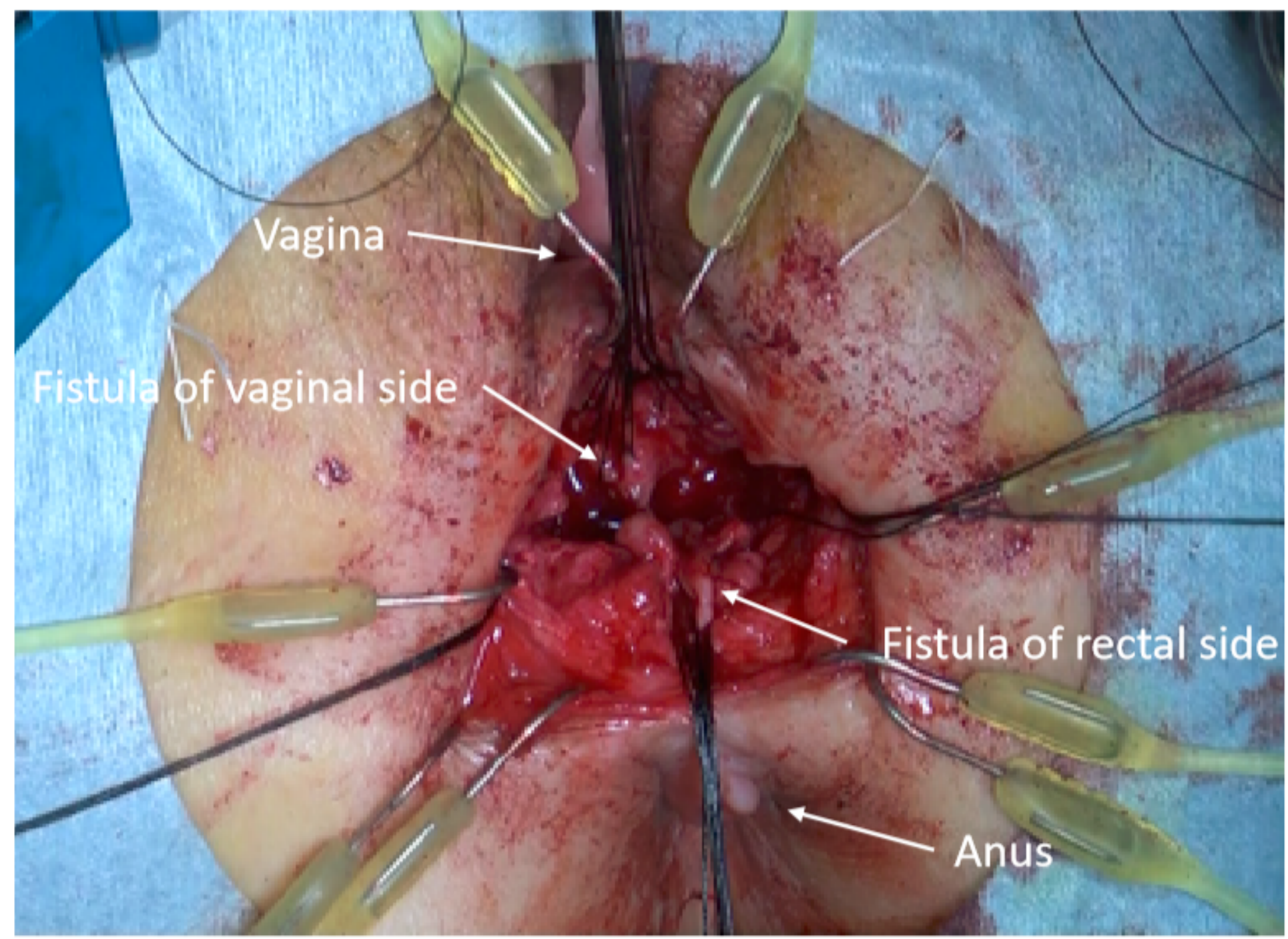

Figure 3

During the dissection of connective tissues between the rectum and vagina, a rectovaginal fistula was released completely. 


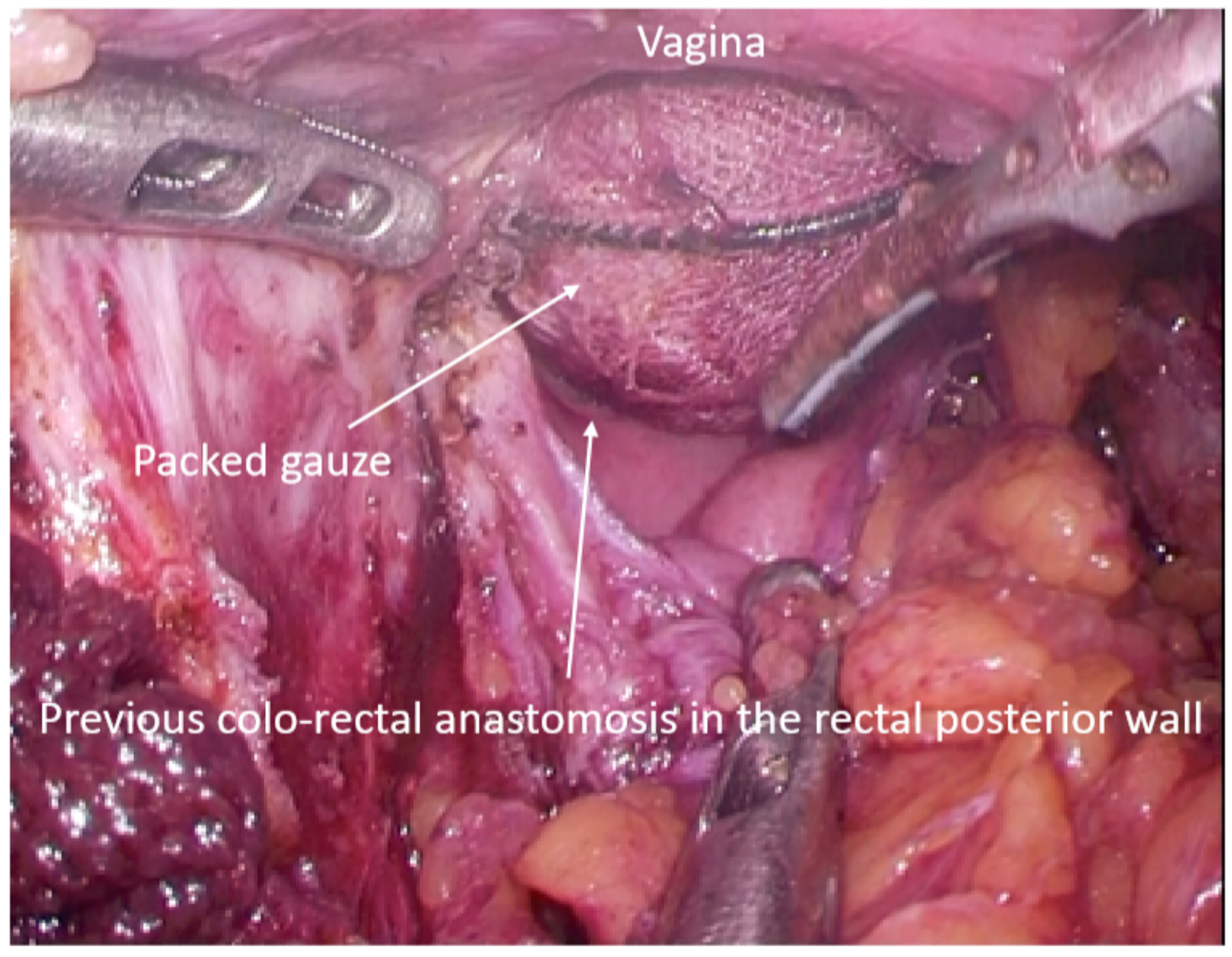

\section{Figure 4}

While resectioning the rectum containing previous anastomosis, a moist gauze was packed in opened perineal space for maintaining pneumoperitoneum. 


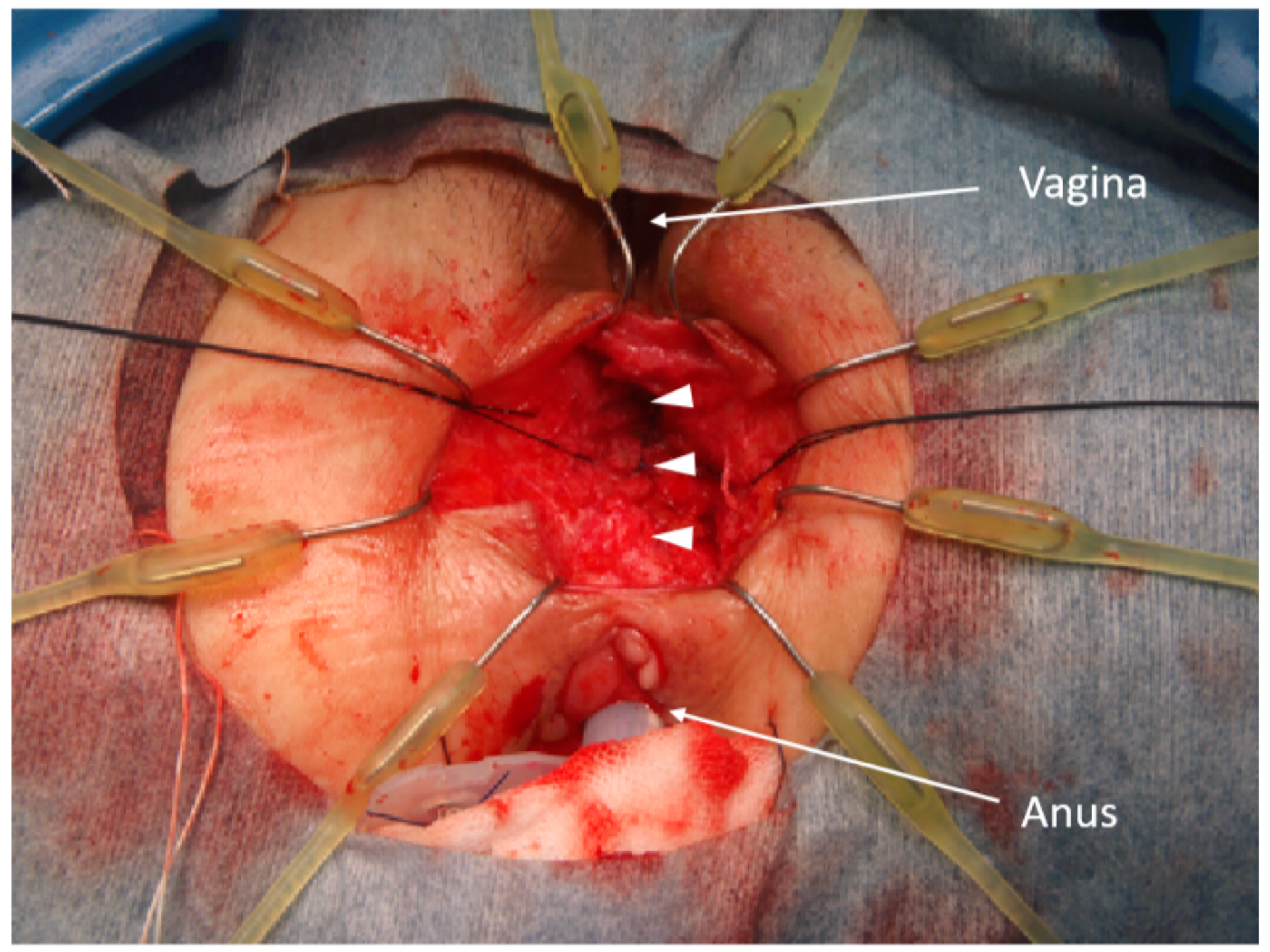

\section{Figure 5}

The innermost portion of the dissected connective tissue was sutured (arrow heads). 